\title{
Protocole d'optimisation et de validation de la qualité de l'image en fonction de la dose (DAP) sur le système Cone Beam CS9300
}

\author{
Thomas Fortin, Stéphane Alric \\ UFR d'Odontologie, Département de Chirurgie buccale, Lyon, France \\ thomas.fortin@univ-lyon1.fr
}

Le projet SEDENTEXCT (www.sedentexct.eu), soutenu par le septième programme-cadre de la Communauté Européenne de l'Energie Atomique (Euratom) a pour but la création de recommandations. Il a permis de dégager des pistes de réflexion pour l'élaboration de projets de recherche pour l'optimisation de l'utilisation de la technologie Cone Beam en Médecine bucco-dentaire, notamment pour la définition des constantes radiologiques utilisées lors de l'examen.

Le but de ces études sur tête de cadavre est d'optimiser certains paramètres radiologiques $(\mathrm{kV}, \mathrm{mA}$ et FoV pour Field of View) pour obtenir la meilleure qualité d'image, en rapport à des tâches diagnostiques, avec pour objectif le principe ALARA (As Low As Reasonably Achievable).

Matériel et méthodes

Nous avons utilisé le système Cone Beam CS9300 (Carestream, Marne-la-Vallée, France), avec QA PASS et tests d'acceptation, ainsi qu'une tête humaine de corpulence moyenne.

Trois étapes distinctes ont été étudiées :

1. Optimisation des $\mathrm{kVp}$ (kilovoltage) et validation sur tête de cadavre, à DAP constant (mGy.cm²). Revues effectuées par des praticiens dentaires, des radiologues et des ORL.

2. Pour un kilovoltage optimisé $(\mathrm{kVp})$, recherche de l'optimisation des milliampères $(\mathrm{mA})$, et validation sur tête de cadavre. Cette étape concerne les 4 spécialités cliniques citées dans la section 3 . Revues effectuées par des praticiens dentaires, des radiologues et des ORL.

3. A $\mathrm{kVp}$ et $\mathrm{mA}$ optimisés, validation clinique basée sur une analyse statistique de cas cliniques. Revues effectuées par des praticiens dentaires, des radiologues et des ORL.

Paramètres évalués de Qualité Image

La qualité d'image sera évaluée pour l'implantologie en utilisant une FoV 5x5 rapide (HS :12s de scan, taille de voxel $=200 u m$ ), utilisation des FoV 10x5 et 10x1.

Conclusion

Sur le plan technique, en laboratoire on note une légère amélioration en baissant les KV (donc en augmentant les $\mathrm{mA}$ ) : un peu plus de contraste image et un peu moins de bruit. Mais on ne peut pas - trop - baisser les KV $(70,65,60 \ldots)$ car l'énergie pourrait ne plus être suffisante pour traverser les tissus denses.

L'évaluation des images par les praticiens montre que la réduction de la dose ne diminue pas la qualité des informations données par l'image.

Il semble tout à fait raisonnable, dans le cadre du respect du principe ALARA, d'utiliser en implantologie des $\mathrm{KV}$ et des $\mathrm{mA}$ relativement faibles, ce qui permettrait d'avoir une irradiation inférieure à celle de la panoramique dentaire. Ceci plaide pour fournir aux praticiens des machines permettent un réglage raisonné des constantes radiologiques.

Il restera à valider les valeurs trouvées dans cette étude sur un groupe de patients. 